\title{
Spontaneous bowel perforation in intestinal scleroderma: first report of a non-fatal case
}

\author{
M. I. V. JAYSON \\ M.D., M.R.C.P. \\ J. Gough \\ M.D., M.R.C.Path.
}

\author{
P. R. SAlmon \\ M.B., M.R.C.P. \\ T. POLINESS \\ M.B., F.R.C.S.
}

\author{
R. L. BISHTON \\ M.D., F.C.Path. \\ Departments of Medicine and Pathology, University of Bristol, \\ Royal National Hospital for Rheumatic Diseases and Royal United Hospital, Bath
}

SCLERODERMA may involve almost any part of the alimentary tract. We describe here a patient with systemic sclerosis and perforation of the sigmoid colon. This is the first report of successful treatment of this complication.

\section{Case report}

The patient was a 35-year-old housewife who developed Raynaud's phenomenon in 1965 . Two years later, when she was admitted into hospital for treatment of broncho-pneumonia, early changes of scleroderma were noted in the hands and face. In May 1969 she developed severe proximal muscle weakness and it was found that the skin changes had considerably extended to involve the hands, forearms, face and anterior chest wall. She also developed dysphagia, abdominal colic, diarrhoea and weightloss. There was mild pyrexia up to $100^{\circ} \mathrm{F}$. Examination showed marked swelling and tenderness of the proximal limb muscles and a provisional diagnosis of myositis associated with scleroderma (Rodnan \& Medsger, 1966) was made. This was confirmed by an elevated creatine phosphokinase ( $>10 \mu \mathrm{M} / \mathrm{ml} / \mathrm{hr}$; normal $<1.5 \mu \mathrm{M} / \mathrm{ml} / \mathrm{hr}$ ). Small-bowel barium-meal examination revealed delayed transit with dilated loops of small intestine and multiple fluid levels. There was no steatorrhoea. These changes were thought to be due to scleroderma involving the small intestine with replacement fibrosis diminishing bowel motility, and secondary bacterial colonization. The small-bowel infection was confirmed by finding increased urinary indoxyl sulphate excretion.

She failed to respond to conservative treatment and was therefore given Synacthen $1 \mathrm{mg}$ intra-

Requests for reprints: Dr M. Jayson, Royal National Hospital for Rheumatic Diseases, Bath. muscularly daily and with this muscular power gradually returned to normal. When she recovered from the episode of muscular weakness she was treated with ampicillin and her bowel symptoms improved dramatically. The motions returned to normal frequency and appearance and the urinary indoxyl sulphate excretion fell sharply. Subsequently, she developed an infection of the pulp of the fourth left finger which progressed despite vigorous conservative therapy and eventually amputation was necessary.

In January she was admitted with a 6-hr history of severe lower abdominal pain with nausea and vomiting. Examination suggested peritonitis and a laparotomy was performed (T.P.). A moderate amount of turbid green fluid was found in the peritoneal space and a perforation at the rectosigmoid junction was noted $2 \mathrm{~cm}$ above the peritoneal reflection through a $5-\mathrm{mm}$ diameter gangrenous patch. The sigmoid colon and upper rectum were mobilized and some $18 \mathrm{~cm}$ of colon were resected including the perforation. The rectal stump was closed and a left iliac colostomy was performed. She made an uneventful postoperative recovery.

\section{Pathology}

The specimen consisted of $18 \mathrm{~cm}$ of colon (Fig. 1). Six centimeters from the distal resection edge there was a perforation and the serosa in this region was covered by fibrinous exudate. The bowel wall was necrotic at the point of perforation but was thickened for about $2.5 \mathrm{~cm}$ on the proximal side of this. On both the proximal and the distal sides of the perforation was a sacculation, each being about $3 \mathrm{~cm}$ in diameter. The walls of the sacculations were thin and lined by flat mucosa. The proximal $8 \mathrm{~cm}$ and 


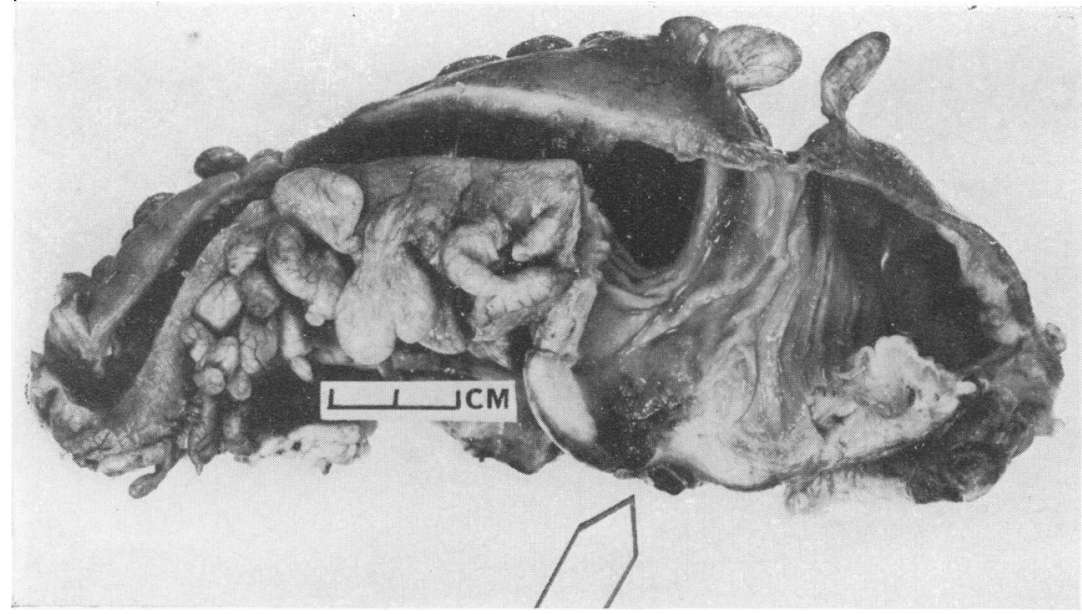

FIG. 1. Resected specimen of colon showing fibrosis of the wall with a perforated ulcer (arrow). A sacculation is present on each side of the ulcer.

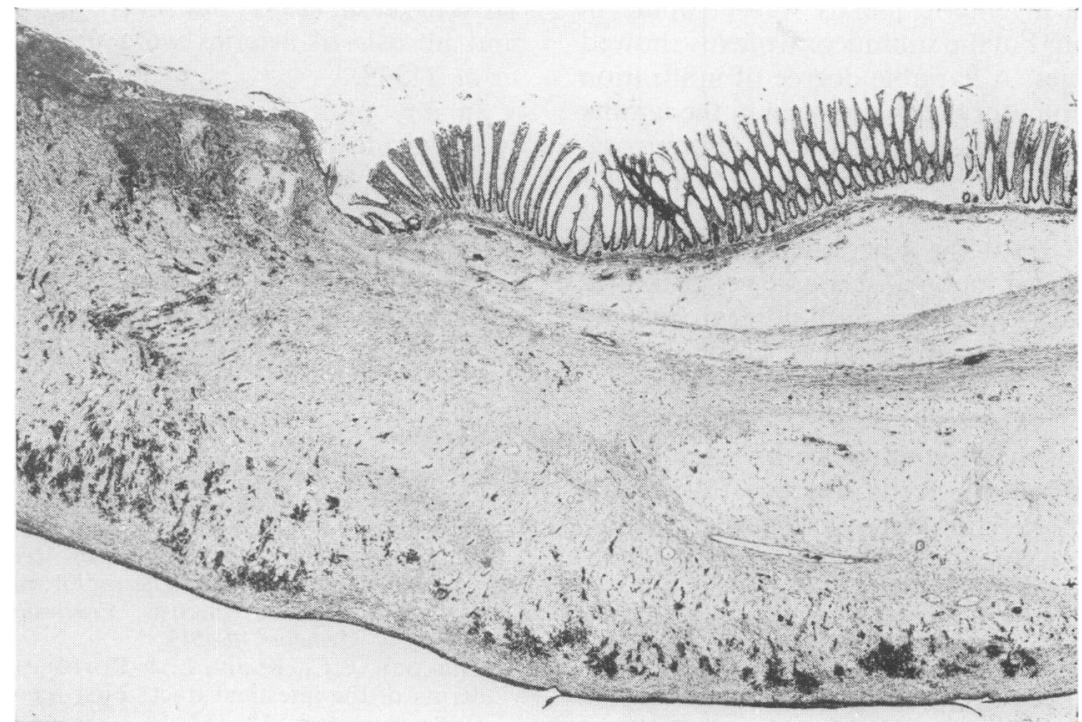

FIG. 2. Section through ulcer, showing fibrosis of the bowel wall. (H and E, $\times 17)$.

distal $2 \mathrm{~cm}$ of the specimen appeared macroscopically normal.

Microscopic examination showed that the perforation had appeared at the site of a chronic ulcer (Fig. 2), this being lined with successive layers of acute inflammatory exudate, necrotic granulation tissue, and healthy granulation tissue. In the floor of the ulcer an artery showed endarteritis obliterans, but there was no evidence of arteritis elsewhere.

The bowel wall in the region of the ulcer showed almost complete replacement of the muscularis externa by fibrous tissue which was moderately cellular. The collagen fibres were generally fine and only occasionally showed the hyaline thickening commonly seen in the cutaneous component of systemic sclerosis. The walls of the sacculations were uniformly thin. The muscle coat of the distal sacculation was thin but not necrotic while the muscularis externa of the proximal sacculation was completely replaced by fibrous tissue (Fig 3). In places the muscularis mucosae was moderately atrophic. In both the area of perforation and in the 


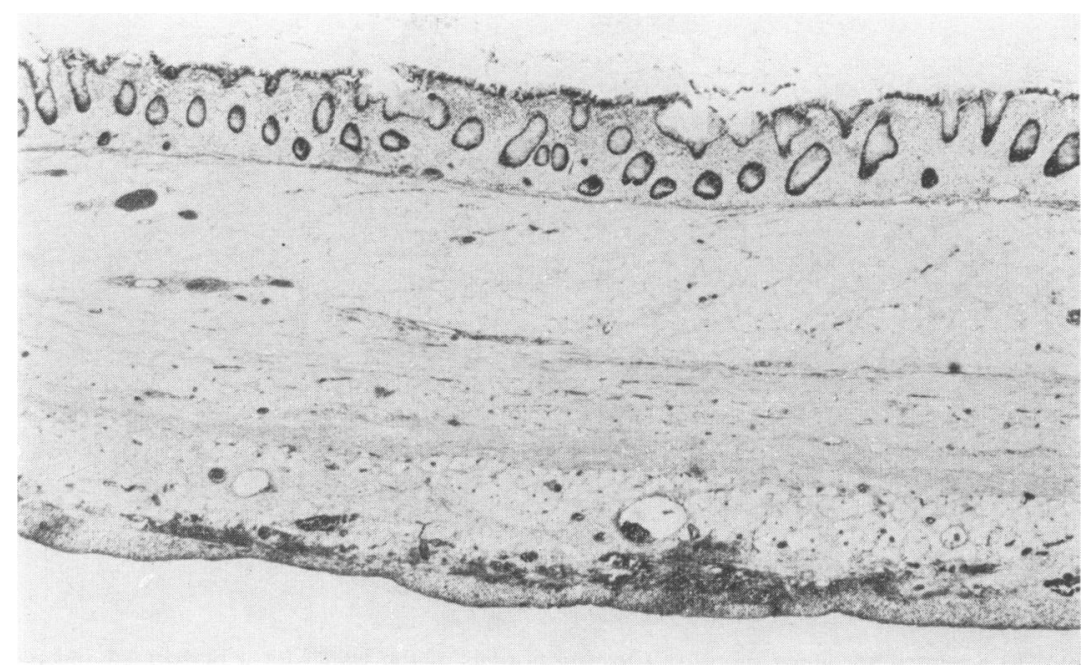

Fig. 3. Proximal sacculation. The miscularis externa is replaced by fibrous tissue (H and E, $\times 24)$.

sacculations the myenteric plexus was atrophic in the fibrotic regions but the submucosal plexus showed less severe changes. A variable degree of infiltration by chronic inflammatory cells was seen in the lamina propria but there was no fibrosis of the submucosa. Sections stained for elastic fibres showed no additional abnormalities.

Bowel remote from the perforated area and the sacculations showed only a secondary peritonitis, indicating that the primary pathological changes were restricted to an $8 \mathrm{~cm}$ length of colon.

\section{Discussion}

Involvement of the large intestine by scleroderma has been described by Kemp-Harper (1953) and Meszaros (1959). The sclerodermatous process involves the muscle coats with weakness and subsequent ballooning of the wall but perforation appears to be a rare complication. Lushbaugh, Rubin \& Rothman (1948), Hoskins et al. (1967) and Nash \& Fountain (1968) each reported one case but in none did the patient survive.

Endarteritis obliterans is often found in the bases of chronic ulcers such as peptic ulcers and in addition no arterial abnormalities were found in the present specimen remote from the ulcer. The vascular chenges are therefore regarded as secondary and not primarily responsible for the ulceration. No vascular lesions were found in the case reported by
Hoskins et al. (1962) but severe intimal proliferation and fibrosis of arteries were found by Lushbaugh et al. (1948).

In any patient with systemic sclerosis who develops acute peritonitis, perforation of an involved segment of bowel should te considered.

\section{Acknowledgments}

We thank Dr A. St. J. Dixon and Mr K. Lloyd-Williams for permission to publish details of this case. One of us (M.I.V.J.) is in receipt of a Research Grant from the Medical Research Council.

\section{References}

Hoskins, L.C., Norris, H.T., Gottlieb, L.S. \& ZAMCheck, N. (1962) Functional and morphological alterations of the gastrointestinal tract in progressive systemic sclerosis (scleroderma). American Journal of Medicine, 33, 459.

KEMP-HARPER, R.A. (1953) The radiological manifestations of diffuse systemic sclerosis. Proceedings of the Royal Society of Medicine, 46, 512.

Lushbaugh, C.C., Rubin, L. \& Rothman, S. (1948) Scleroderma of the intestinal tract: First report of a fatal case. Gastroenterology, 11, 382.

Meszaros, W.T. (1959) The colon in systemic sclerosis (scleroderma). American Journal of Roentgenology, 82, 1000.

Nash, A.G. \& Fountain, R. (1968). Surgical presentation of systemic sclerosis in the small intestine. British Journal of Surgery, 55, 667.

Rodnan, G.P. \& Medsger, T.A. (1966) Musculo-skeletal involvement in progressive systemic sclerosis. Bulletin of Rheumatic Diseases, 17, 419. 